\title{
INBREEDING AND THE FUNDAMENTAL THEOREM OF NATURAL SELECTION
}

\author{
S. P. H. MANDEL \\ Mathematics-Statistics Unit, Division of Research in Epidemiology \\ and Communications Science \\ and \\ I. BARRAI \\ Human Genetics Unit, Division of Family Health, World Health Organization, \\ Geneva, Switzerland
}

Received 6.iii.72

\section{INTRODUGTION}

FrsHeR's fundamental theorem of natural selection (Fisher, 1930) has been the subject of considerable discussion in recent years (Edwards, 1967; Li, 1967a, b; Turner, 1967; Mandel, 1968; Ewens, 1969; Arunachalam, 1970; Karlin and Feldman, 1970). In one of its forms, when applied in the discrete generation model to the situation of a single genetic locus under constant genotypic selection in a random mating population, the theorem states that the average fitness does not decrease (Karlin and Feldman, 1970). Much of the discussion has been concerned with the identification of the most general conditions under which the theorem continues to hold. It is interesting to note that the average fitness can decrease in populations in which there is inbreeding and that, therefore, the theorem is no longer valid in that situation. This has been pointed out by Turner (1970) in a recent article. The purpose of this note is to examine some of the properties of the change in average fitness as a function of the gene frequency when there is inbreeding in the case of a genetical system consisting of two alleles at a single locus with constant differential viability parameters.

\section{Derivation of Results}

If $a, h$ and $b$ represent the selection parameters corresponding to genotypes $A A, A a$ and $a a$ respectively, and $p$ is the gene frequency of allele $A$ in a specified generation $(q=1-p$ is therefore the gene frequency of allele $a)$, then it can be shown that the relationship between $p$ and $p^{\prime}$, the gene frequency of allele $A$ in the next generation, can be written in the following forms:

and

$$
p^{\prime}-p=\frac{p q\{(a-h)(p+F q)+(h-b)(q+F p)\}}{a p(p+F q)+2 h p q(1-F)+b q(q+F p)}
$$

where $F$ is the coefficient of inbreeding and

$$
P_{F}=\frac{(h-b)-(h-a) F}{\{(h-a)+(h-b)\}(1-F)}
$$


is the value of $p$ for which the difference $p^{\prime}-p$ vanishes, that is to say $P_{F}$ is the unique polymorphic equilibrium gene frequency whenever $0<P_{F}<1$. It is clear that (1) and (2) reduce to the classical forms for the random mating situation in the absence of inbreeding if we set $F=0$.

The average fitness of the population is given by the expression in the denominator of (1) and (2), and, denoting this expression by $V$, we can write

$$
V=a p^{2}+2 h p q+b q^{2}+F p q(a-2 h+b) .
$$

It is well known (see e.g. Mandel, 1968) that, in the absence of inbreeding with random mating $(F=0)$,

$$
V^{\prime}-V=\frac{1}{V^{2}}\left\{p(a p+h q-V)^{2}+q(h p+b q-V)^{2}\right\}(V+a p+b q) \geqq 0
$$

where $V^{\prime}$ is the average fitness of the population in the next generation. We will show now that, when there is inbreeding, the expression $V^{\prime}-V$ can in fact be negative. The general expression for the difference $V^{\prime}-V$ is a complex one, and it is convenient to look at the situation in the neighbourhood of $p=0$, i.e. for small values of $p$, since this enables us to make linear approximations. Thus we can write, for small values of $p$,

$$
V=2(h-b) p+b+F p(a-2 h+b)
$$

and hence

$$
V^{\prime}-V=\{(h-b)+F(a-2 h+b)\}\left(p^{\prime}-p\right) .
$$

Now from (1) we obtain the approximation

$$
p^{\prime}-p=\frac{p}{b}\{(a-h) F+h-b\}
$$

so that we have, correct to terms of the first order of magnitude in $p$,

$$
V^{\prime}-V=\frac{p}{b}\{(a-h) F+h-b\}\{2(h-b)+F(a-2 h+b)\} .
$$

Note that for $F=0$ we obtain the random mating solution

$$
V^{\prime}-V=\frac{2 p}{b}(h-b)^{2} \geqq 0
$$

but that when $F \neq 0$ we can obtain a decrease in the value of $V$ from one generation to the next. For example, if we take the model $a=1, h=6$, $b=4$ and $F=\frac{1}{2}$ we obtain $V^{\prime}-V=\frac{-p}{16}$.

We observed empirically on the basis of computer simulations of stable systems that it was possible for the mean fitness to decrease, but that this could only occur when the initial frequency of the less fit allele was greater than its equilibrium value. This observation led to some interesting inequalities between the equilibrium gene frequencies $P_{F}$ and the gene frequency for which the value of the mean fitness is stationary. Denoting the 
latter by $\hat{P}$ and setting the derivative of $V$ with respect to $p$ equal to zero we obtain

$$
\hat{P}=\frac{P_{0}-\frac{1}{2} F}{1-F}
$$

where $P_{0}$ is the equilibrium gene frequency in the absence of inbreeding.

Moreover, it follows from (3) that

$$
P_{F}=\frac{P_{0}-Q_{0} F}{1-F}
$$

and finally, eliminating $P_{0}$ from (11) and (12) we have

$$
P_{F}=(1+F) \hat{P}-\frac{1}{2} F \text {. }
$$

Now let us consider some special cases. Firstly it follows from the condition $0<P_{F}<1$ that a realisable equilibrium exists if and only if

or

$$
\left.\begin{array}{l}
h-a>F(h-b) \\
h-b>F(h-a)
\end{array}\right\}
$$

and from (2) it follows that the equilibrium characterised by (14) is stable while that characterised by (15) is unstable (Barrai and Mandel, 1971). Furthermore, the inequalities (14) imply the necessary condition

$$
(h-a+h-b)(1-F)>0
$$

which in turn implies the necessity of heterozygote advantage for the existence of a stable equilibrium, even when inbreeding is present. Thus, in studying stable systems, we can assume without loss of generality, that $h>a>b$ so that, $q$ being the gene frequency of the less fit allele, we have $Q_{0}<\frac{1}{2}$.

Now, we have from (11)

$$
\hat{Q}-Q_{0}=\frac{Q_{0}-\frac{1}{2} F}{1-F}-Q_{0}=\frac{F}{1-F}\left(Q_{0}-\frac{1}{2}\right)<0
$$

and from (13) we have

$$
Q_{F}-\hat{Q}=F\left(\hat{Q}-\frac{1}{2}\right)<0
$$

so that we have $Q_{F}<\hat{Q}<Q_{0}<\frac{1}{2}$. It follows from

$$
\frac{d^{2} V}{d p^{2}}=2(a-2 h+b)(1-F)<0,
$$

under our earlier assumption, that the stationary value of $V$ corresponds in this case to a maximum. Since it follows from (2) that the approach of the gene frequency towards its equilibrium value is monotonic, it follows also that if the initial gene frequency of the less fit allele is less than the equilibrium value then the mean fitness will increase monotonically as the population approaches equilibrium, but that this will no longer be the case if the initial gene frequency of the less fit allele exceeds the equilibrium value. 
As an example, if we take the model

$$
a=0 \cdot 9, h=1 \cdot 0, b=0 \cdot 8, F=0 \cdot 1
$$

we find that

$$
Q_{F}=0.2963, \hat{Q}=0.3148, Q_{0}=0.3333
$$

and starting with an initial gene frequency of $q_{0}=\frac{8}{15}$ the gene frequencies and the values of the mean fitness are shown in the table below.

\section{TABLE}

Gene frequencies (q) and mean fitness values in one hundred successive generations starting from initial frequency

$$
q_{0}=\frac{8}{15}
$$

\begin{tabular}{|c|c|c|}
\hline $\begin{array}{c}\text { Generation } \\
\text { number }\end{array}$ & $\begin{array}{c}\text { Gene frequency } \\
(q)\end{array}$ & $\begin{array}{l}\text { Mean fitness } \\
(V)\end{array}$ \\
\hline 0 & 0.53333 & 0.91387 \\
\hline 5 & 0.45817 & 0.92018 \\
\hline 10 & 0.40776 & 0.92398 \\
\hline 15 & 0.37400 & 0.92562 \\
\hline 20 & 0.35109 & 0.92632 \\
\hline 25 & 0.33529 & 0.92661 \\
\hline 30 & 0.32424 & 0.92672 \\
\hline 35 & 0.31642 & 0.92676 \\
\hline 40 & $0 \cdot 31085$ & 0.92676 \\
\hline 45 & 0.30685 & 0.92674 \\
\hline 50 & 0.30397 & 0.92673 \\
\hline 55 & 0.30188 & 0.92672 \\
\hline 60 & 0.30037 & 0.92670 \\
\hline 65 & $0 \cdot 29926$ & 0.92670 \\
\hline 70 & $0 \cdot 29846$ & 0.92669 \\
\hline 75 & $0 \cdot 29788$ & 0.92668 \\
\hline 80 & $0 \cdot 29745$ & 0.92668 \\
\hline 85 & 0.29714 & 0.92668 \\
\hline 90 & $0 \cdot 29691$ & 0.92667 \\
\hline 95 & $0 \cdot 29675$ & 0.92667 \\
\hline 100 & $0 \cdot 29662$ & 0.92667 \\
\hline
\end{tabular}

Parameter values $a=0 \cdot 9, h=1 \cdot 0, b=0 \cdot 8, F=0 \cdot 1$

\section{Summary}

1. Some of the theoretical and numerical properties are examined of a genetical system with inbreeding at a single locus with two alleles and fixed viability parameters, with particular reference to the fundamental theorem of natural selection.

2. It is noted in particular (Turner, 1970) that in the presence of inbreeding the average fitness of the population can decrease under certain conditions and an illustrative numerical example is given.

\section{REFERENGES}

arunachalam, B. 1970. Fundamental theorem of natural selection in two loci. Ann. Hum. Genet., Lond., 34, 195-199.

BARRAI, I., AND MANDEL, S. P. H. 1971. Equilibrium under inbreeding in balanced polymorphisms. Bull. World Health Org., 45, 201-208. 
EDWARDS, A. W. F. 1967. Fundamental theorem of natural selection. Nature, 215, 537-538. EwENs, w. J. 1969. Mean fitness increases when fitnesses are additive. Nature, 211, 1076. FISHER, R. A. 1930. The Genetical Theory of Natural Selection. Oxford University Press. KARLIN, S., AND FELDMAN, M. W. 1970. Convergence to equilibrium of the two locus additive viability model. 7. Appl. Prob., 7, 262-271.

LI, c. c. 1967a. Genetic equilibrium under selection. Biometrics, 23, 397-484.

LI, C. C. 1967b. Fundamental theorem of natural selection. Nature, 214, 505-506.

MANDEL, S. P. H. 1968. Fundamental theorem of natural selection. Nature, 220, 1251-1252.

TURNER, J. R. G. 1967. Fundamental theorem of natural selection. Nature, 215, 1080.

TURNER, J. R. G. 1970. Changes in mean fitness under natural selection. In Mathematical Topics in Population Genetics (ed. Kojima). Springer-Verlag. 